\title{
Distributed Partial Clustering
}

\author{
Sudipto Guha \\ University of Pennsylvania \\ Philadelphia, PA 19104, United States \\ sudipto@cis.upenn.edu
}

\author{
Yi Li \\ Nanyang Technological University \\ Singapore \\ yili@ntu.edu.sg
}

\author{
Qin Zhang \\ Indiana University Bloomington \\ Bloomington, IN 47401, United States \\ qzhangcs@indiana.edu
}

\begin{abstract}
Recent years have witnessed an increasing popularity of algorithm design for distributed data, largely due to the fact that massive datasets are often collected and stored in different locations. In the distributed setting communication typically dominates the query processing time. Thus it becomes crucial to design communication efficient algorithms for queries on distributed data. Simultaneously, it has been widely recognized that partial optimizations, where we are allowed to disregard a small part of the data, provide us significantly better solutions. The motivation for disregarded points often arise from noise and other phenomena that are pervasive in large data scenarios.

In this paper we focus on partial clustering problems, $k$-center, $k$-median and $k$-means, in the distributed model, and provide algorithms with communication sublinear of the input size. As a consequence we develop the first algorithms for the partial $k$-median and means objectives that run in subquadratic running time. We also initiate the study of distributed algorithms for clustering uncertain data, where each data point can possibly fall into multiple locations under certain probability distribution.
\end{abstract}

\section{INTRODUCTION}

The challenge of optimization over large quantities of data has brought communication efficient distributed algorithms to the fore. From the perspective of optimization, it has also become clear that partial optimizations, where we are allowed to disregard a small part of the input, enable us to provide significantly better optimization solutions compared with those which are forced to account for the whole input. While several algorithms for distributed clustering have been proposed, partial optimizations for clustering problems, introduced by Charikar et al. [4], have not received as much attention. While the results of Chen [6] improve the approximation ratios, the running time of the $k$-median and $k$-means versions have not been improved and the (at least) quadratic running times have remained as a barrier.

In this paper we study partial clustering under the standard $(k, t)$ median/means/center objective functions, where $k$ is the number of

\footnotetext{
Sudipto Guha was supported in part by NSF award 1546151. Qin Zhang was supported in part by NSF CCF-1525024 and IIS-1633215.

Permission to make digital or hard copies of all or part of this work for personal or classroom use is granted without fee provided that copies are not made or distributed for profit or commercial advantage and that copies bear this notice and the full citation on the first page. Copyrights for components of this work owned by others than the author(s) must be honored. Abstracting with credit is permitted. To copy otherwise, or republish, to post on servers or to redistribute to lists, requires prior specific permission and/or a fee. Request permissions from permissions@acm.org.

SPAA '17, fuly 24-26, 2017, Washington DC, USA

(C) 2017 Copyright held by the owner/author(s). Publication rights licensed to Association for Computing Machinery.

ACM ISBN 978-1-4503-4593-4/17/07 ..\$15.00

https://doi.org/10.1145/3087556.3087568
}

centers we can use and $t$ is the maximum number of points we can ignore. In the distributed setting, let $s$ denote the number of sites. The $(k, t)$-center problem has recently been studied by Malkomes et al. [19], who gave a 2-round $O(1)$-approximation algorithm with $\tilde{O}(s k+s t)$ bits of communication ${ }^{1}$, assuming that each point can be encoded in $O(1)$ bits. In fact, we observe that results from streaming algorithms [14] can in fact provide us 1-round $O(1)$-approximation algorithms with $\tilde{O}(s k+s t)$ bits of communication for $(k, t)$-center, $(k, t)$-median, and $(k, t)$-means. However, in many scenarios of interest, we have $n>t \gg k$ and $t \gg s$. Thus the $s t$ term generates a significant communication burden. In this paper we reduce $\tilde{O}(s t)$ to $\tilde{O}(t)$ for the $(k, t)$-center problem, as well as for $(k, t)$-median and $(k, t)$-means problems and unify their treatment. We also provide the first subquadratic algorithms for median and means version of this problem.

Large data sets often have erroneous values. Stochastic optimization has recently attracted a lot of attention in the field of databases, and has substantiated as a subfield called 'uncertain/probabilistic databases' (see, e.g., [20]). For the clustering problem, a method of choice is to first model the underlying uncertainty and then cluster the uncertain data. Clustering under uncertainty has been studied in centralized models $[8,15]$, but the algorithms proposed therein do not consider communication costs. Note that it typically requires significantly more communication to communicate a distribution (for an uncertain point) than a deterministic point, and thus black box adaptations of centralized algorithms do not work well in the distributed setting. In this paper we propose communicationefficient distributed algorithms for handling both data uncertainty and partial clustering. To the best of our knowledge neither distributed clustering of uncertain data nor partial clustering of uncertain data has been studied. We note that both problems are fairly natural, and likely to be increasingly useful as distributed cloud computing becomes commonplace.

Models and Problems. We study the clustering problems in the coordinator model, in which there are $s$ sites and one central coordinator, who are connected by a star communication network with the coordinator at the center. However, direct communication between sites can be simulated by routing via the coordinator, which at most doubles the communication. The computation is in terms of rounds. At each round, the coordinator sends a message (could be an empty message) to each site and every site sends a message (could be an empty message) back to the coordinator. The coordinator outputs the answer at the end. The input $\mathbb{A}$ is partitioned into $\left(\mathbb{A}_{1}, \ldots, \mathbb{A}_{s}\right)$ among the $s$ sites. Let $n_{i}=\left|\mathbb{A}_{i}\right|$, and $n=|\mathbb{A}|=\sum_{i \in[s]} n_{i}$ be the total input size.

We will consider clustering over a graph with $n$ nodes and an oracle distance function $d(\cdot, \cdot)$. An easy example of such is points

\footnotetext{
${ }^{1}$ We hide poly $\log n$ factors in the $\tilde{O}$ notation.
} 
in Euclidean space. More complicated examples correspond to documents and images represented in a feature space and the distance function is computed via a kernel. We now give the definitions of $(k, t)$-center/median/means.

Definition $1.1((k, t)$-center,median,means). Let $\mathbb{A}$ be a set of $n$ points and $k, t$ are integer parameters $(1 \leq k \leq n, 0 \leq t \leq n)$. In the $(k, t)$-median problem we want to compute

$$
\min _{K, \mathbb{O} \subseteq \mathbb{A}} \sum_{p \in \mathbb{A} \mid \mathbb{O}} d(p, K) \quad \text { subject to }|K| \leq k \text { and }|\mathbb{O}| \leq t,
$$

where $d(p, K)=\min _{x \in K} d(p, x)$. We typically call $K$ the centers and (1) the outliers. In the $(k, t)$-means and the $(k, t)$-center problem we replace the objective function $\sum_{p \in \mathbb{A} \mid \mathcal{O}} d(p, K)$ with $\sum_{p \in \mathbb{A} \backslash \mathbb{O}} d^{2}(p, K)$ and $\max _{p \in \mathbb{A} \mid \mathcal{O}} d(p, K)$ respectively.

In the definition above, we assume that centers are chosen from the input points. In the Eucldiean space, such restriction will only affect the approximation by a factor of 2 .

For the uncertain data, we follow the assigned clustering introduced in [8]. Let $\mathcal{P}$ be a finite set of points in a metric space. There are $n$ input nodes $\mathbb{A}$, where node $j$ follows an independent distribution $\mathcal{D}_{j}$ over $\mathcal{P}$. Each site $i$ knows the distributions $\mathcal{D}_{j}$ associated with the nodes $j \in \mathbb{A}_{i}$.

Definition 1.2 (Clustering Uncertain Data). In clustering with uncertainty, the output is a subset $K \subseteq \mathcal{P}$ of size $k$ (centers), a subset $\mathbb{O} \subseteq \mathcal{P}$ of size at most $t$ (ignored points), as well as a mapping $\pi: \mathbb{A} \rightarrow K$. In every realization $\sigma: \mathbb{A} \rightarrow \mathcal{P}$ of the values of the input nodes, node $j \in \mathbb{A}$ (now realized as $\sigma(j) \in \mathcal{P}$ ) is assigned to the same center $\pi(j) \in K$. In uncertain $(k, t)$-median, the goal is to minimize the expected cost

$$
\underset{\sigma \sim \prod_{j \in \mathbb{A}}}{\mathbb{E}}\left[\sum_{j} d(\sigma(j), \pi(j))\right]=\sum_{j \in \mathbb{A}|\mathcal{O}| \mathcal{O}} \underset{\sigma \sim \mathcal{D}_{j}}{\mathbb{E}}[d(\sigma(j), \pi(j))] .
$$

The definition of uncertain $(k, t)$-means is basically the same as uncertain $(k, t)$-median, except that we replace the objective function (1) with $\sum_{j \in \mathbb{A} \mid \mathcal{O}} \mathbb{E}_{\sigma \sim \mathcal{D}_{j}}\left[d^{2}(\sigma(j), \pi(j))\right]$. For uncertain $(k, t)$-center, we have two objectives:

$$
\begin{array}{r}
\max _{j \in \mathbb{A} \mid \mathcal{O}}\left(\underset{\sigma \sim \mathcal{D}_{j}}{\mathbb{E}}[d(\sigma(j), \pi(j))]\right) \\
\underset{\sigma \sim \prod_{j} \mathcal{D}_{j}}{\mathbb{E}}\left[\max _{j \in \mathbb{A} \mid \mathcal{O}} d(\sigma(j), \pi(j))\right]
\end{array}
$$

Note that these two objectives are not equivalent, since $\mathbb{E}$ and max do not commute in Equation (3) and we cannot equate it to (2). Equation (2) is in the same spirit as Equation (1), and corresponds to a per point measurement. We term this problem as uncertain $(k, t)$ center-pp. Equation (3) corresponds to a more global measurement and we term this problem as uncertain $(k, t)$-center-g. This version was considered in $[8,15]$.

Our Results. We present our main results in Table 1 and only present the results based on 2 rounds. The full set of our results can be found in Appendix A. We use $T$ to denote the runtime to compute 1-median/means of a node distribution, $B$ to denote the information needed to encode a point, and $I$ to denote the information needed to encode a node in the uncertain data case. In the column of Local Time, the first is the local computation time of all sites, and the second is the local computation time at the coordinator. Observe that the total running time is $\tilde{O}\left(\sum_{i} n_{i}^{2}\right)$, which becomes $\tilde{O}\left(n^{2} / s\right)$ if the partitions are balanced. This shows that we can reduce the running time by distributing the clustering across many sites.

In particular we have obtained the following. All algorithms finish in 2 rounds in the coordinator model. We say a solution is an $(\alpha, \beta)$-approximation if it is a solution of $\operatorname{cost} \alpha C$ while excluding $\beta t$ points, where $C$ is the optimum cost for excluding $t$ points.

(1) We give $(O(1), 1)$-approximation algorithms with $\tilde{O}((s k+t) B)$ communication for the $(k, t)$-median (Section 3$)$ and the $(k, t)$ center (Theorem 4.3) problems. The lower bounds in [5] for the $t=0$ case indicate that these communication costs are tight, if we want to output all the outliers (which our algorithms do), up to logarithmic factors. We also give an $(O(1+1 / \epsilon), 1+\epsilon)$ approximation algorithm with $\tilde{O}((s k+t) B)$ communication for the $(k, t)$-median (with better running time) and the $(k, t)$-means (Theorem 3.6) problems.

(2) We show that for $(k, t)$-median/means and $(k, t)$-center-pp the above results are achievable even on uncertain data (Theorem 5.6). For uncertain $(k, t)$-center-g we obtain an $(O(1+$ $1 / \epsilon), 1+\epsilon)$-approximation algorithm with $\tilde{O}(s k B+t I+s \log \Delta)$ communication, where $I$ is the information to encode the distribution of an uncertain point, and $\Delta$ is the ratio between the maximum pairwise distance and the minimum pairwise distance in the dataset (Theorem 5.14).

Our results for the $(k, t)$-center problem improves that in [19]. And as far as we are concerned, our results on distributed $(k, t)$ median/means and of uncertain input are the first of their kinds. Our results for distributed $(k, t)$-median or means also lead to subquadratic time constant factor approximation centralized algorithms, which have been left open for many years.

Technical Overview. The high level idea of our algorithms is fairly natural: Each site first performs a preclustering, i.e., it computes some local solution on its own dataset. Then each site sends the centers of the local solution, number of attached points to each center and the ignored points to the coordinator, who will then solve the induced weighted clustering problem.

A major difficulty is to determine how many points to ignore in the local solution at each site. Certainly for the sake of safety each site can ignore $t$ points and send all ignored $t$ points to the coordinator for a final decision. This would however incur $\Theta(s t)$ bits of communication. To reduce the communication of this part to $O(t)$, we hope to find $\left\{t_{1}, \ldots, t_{s}\right\}$ such that $\sum_{i} t_{i}=t$ and each site $i$ sends a solution with just $t_{i}$ ignored points. At the cost of an extra round of communication, we solve the minimization problem $\sum_{i} f_{i}\left(t_{i}\right)$ subject to $\sum_{i} t_{i}=t$ for convex functions $\left\{f_{i}\right\}$. It is tempting to take $f_{i}\left(t_{i}\right)$ to be the cost of local solution with $t_{i}$ ignored points on site $i$, however, such $f_{i}$ is not necessarily convex. The remedy is to take a lower convex hull of $f_{i}$ instead, which can be shown to have only a mild effect on the solution cost. The convex hull of $t$ points can be found in $O(t \log t)$ time, and we can further reduce the runtime without compromising approximation ratio by computing local solutions on each site for only $\log t$ geometrically increasing values of $t_{i}$. 


\begin{tabular}{|c|c|c|c|c|c|c|}
\hline Objective & Approx. & Centers & Ignored & Rounds & Total Comm. & Local Time \\
\hline median & $\begin{array}{c}O(1) \\
O(1+1 / \epsilon)\end{array}$ & $k$ & $\begin{array}{c}t \\
(1+\epsilon) t\end{array}$ & 2 & $\begin{array}{c}\tilde{O}((s k+t) B) \\
\tilde{O}((s k+t) B)\end{array}$ & $\begin{array}{c}\tilde{O}\left(n_{i}^{2}\right), \tilde{O}\left(k^{2} t^{2}(s k+t)^{3}\right) \\
\tilde{O}\left(n_{i}^{2}\right), \tilde{O}\left((s k+t)^{2}\right)\end{array}$ \\
\hline means & $O(1+1 / \epsilon)$ & $k$ & $(1+\epsilon) t$ & 2 & $\tilde{O}((s k+t) B)$ & $\tilde{O}\left(n_{i}^{2}\right), \tilde{O}\left((s k+t)^{2}\right)$ \\
\hline center & $O(1)$ & $k$ & $t$ & 2 & $\tilde{O}((s k+t) B)$ & $\tilde{O}\left((k+t) n_{i}\right), \tilde{O}\left((s k+t)^{2}\right)$ \\
\hline $\begin{array}{c}\text { uncertain } \\
\text { median/ } \\
\text { means/ } \\
\text { center-pp }\end{array}$ & \multicolumn{7}{|c|}{ as in the regular case above } & $+O\left(n_{i} T\right)$, unchanged \\
\hline center-g & $O(1+1 / \epsilon)$ & $k$ & $(1+\epsilon) t$ & 2 & $\tilde{O}(s k B+t I+s \log \Delta)$ & $\tilde{O}\left(n_{i}^{2} \log \Delta\right), \tilde{O}\left((s k+t)^{2}\right)$ \\
\hline
\end{tabular}

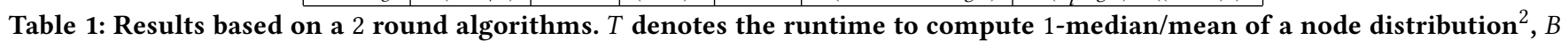
the information encoding a point and $I$ the information encoding a node in the uncertain data case. $\Delta$ is the ratio between the maximum pairwise distance and the minimum pairwise distance in the dataset.

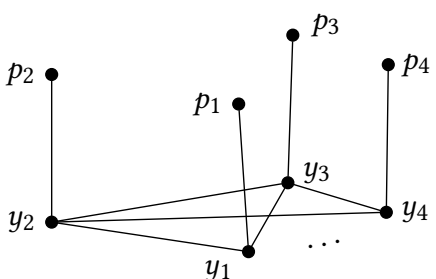

Figure 1: An example of a compressed graph produced

For uncertain data, it is natural to reduce the clustering problems to the deterministic case. To this end, we 'collapse' each node $j$ to its optimal center in $\mathcal{P}$. For instance, for the $(k, t)$-median problem, each node $j$ is 'collapsed' to $y_{j}=\arg \min _{y \in \mathcal{P}} \mathbb{E}_{\sigma}[d(\sigma(j), y)]$, called the 1-median of node $j$. It may be tempting to consider the clustering problem on the set of 1-medians, but the 'collapse' cost is lost, hence we construct a compressed graph $G$ that allows us to keep track of the collapse costs. The graph looks like a clique with tentacles, see Figure 1. The 1-medians form a clique in $G$ with edge weight being the distance in the underlying metric space; for each 1-median $y_{j}$, we add a tentacle (an edge) from $y_{j}$ to a new vertex $p_{j}$ with edge weight being the collapse cost $\mathbb{E}_{\sigma}\left[d\left(\sigma(j), y_{j}\right)\right]$. We manage to show that the original clustering problem is equivalent, up to a constant factor in cost, to the clustering problem on the compressed graph where the facility vertices are 1-medians $\left\{y_{j}\right\}$ and the demand vertices are $\left\{p_{j}\right\}$. Our previous framework for deterministic data is then applied to the compressed graph.

Lastly, for the global center problem with uncertain data, we build upon the approach developed in [15], which uses a truncated distance function $\mathcal{L}_{\tau}(x, y)=\max \{d(x, y)-\tau, 0\}$ instead of the usual metric distance $d(\cdot, \cdot)$. Our algorithm performs a parametric search on $\tau$, and applies our previous framework to solve the global problem using local solutions. Now in the analysis of the approximation ratio we need to relate the optimum solution to the solution with truncated distance function, which is a fairly nontrivial task.

Related Work. In the centralized model, Charikar et al. gives a 3-approximation algorithm for $(k, t)$-center, and an $(O(1), O(1))$ bicriteria algorithm for $(k, t)$-median [4]. This bicriteria was later

\footnotetext{
${ }^{2}$ For a general discrete distribution on $m$ points in Euclidean space with $\mathcal{P}$ be the whole space, $T=O(m)$ [10]; for special distributions such as normal distribution, $T=O(1)$.
}

removed by Chen [6], who designed an $O(1)$-approximation algorithm using $\tilde{O}\left(k^{2}(k+t)^{2} n^{3}\right)$ time. Feldman and Schulman studied the $(k, t)$-median problem with different loss functions using the coreset technique [12].

On uncertain data, Cormode and McGregor considered $k$-center/ median/means where each $\mathcal{D}_{i}$ is a discrete distribution [8]. Guha and Munagala provided a technique to reduce the uncertain $k$ center to the deterministic $k$-median problem [15]. Wang and Zhang studied the special case of $k$-center on the line [21]. We refer the readers to the survey by Aggarwal [1].

Clustering on distributed data has been studied only recently. In the coordinator model, in the $d$-dimensional Euclidean space, Balcan et al. obtained $O(1)$-approximation algorithms with $\tilde{O}((k d+s k) B)$ bits of communication for both $k$-median and $k$-means [2]. Their results on $k$-means were further improved by Liang et al. [18] and Cohen et al. [7]. Chen et al. provided a set lower bounds for these problems [5]. In the MapReduce model, Ene et al. designed several $O(1)$-approximation $O(1)$-round algorithms for the $k$-center and the $k$-median problems [11]. Im and Moseley further studied the partial clustering variant [16], however their algorithms require communication polynomial in $n$. Cormode et al. studied the $k$-center maintenance problem in the distributed data stream model where the coordinator can keep track of the cluster centers at any time step [9].

\section{PRELIMINARIES}

Notation. We use the following notations in this paper.

- $\operatorname{sol}(Z, k, t, d)$ : A solution (computed by an algorithm) to the median/means/center problem on point set $Z$ with at most $k$ centers and at most $t$ outliers, under the distance function $d$;

- $\operatorname{opt}(Z, k, t, d)$ : An optimal solution to the median/means or center problem on point set $Z$ with at most $k$ centers and at most $t$ outliers, under $d$;

- $C_{\text {sol }}(Z, k, t, d)$ : The cost of the solution $\operatorname{sol}(Z, k, t, d)$;

- $C_{\text {opt }}(Z, k, t, d)$ : The cost of the solution opt $(Z, k, t, d)$;

- $\pi(j)$ : The center to which point $j$ is attached.

When $Z$ lies in a metric space and $d$ agrees with the distance function on the metric space, we omit the parameter $d$ in the notations above.

Combining Preclustering Solutions. We review a theorem from [14], which concerns 'combining' local solutions into a global solution. The problems considered in the theorem have no outliers 
$(t=0)$ and lie in a metric space, so we abbreviate the notation $\operatorname{sol}(Z, k, t, d)$ to $\operatorname{sol}(Z, k)$, etc.

Theorem $2.1([14])$. Suppose that $\mathbb{A}=\mathbb{A}_{1} \uplus \cdots \uplus \mathbb{A}_{s}$ (disjoint union) and $\left\{\operatorname{sol}\left(\mathbb{A}_{i}, k\right)\right\}$ are the preclustering solutions at sites. Let $\mathbb{M}=\{\pi(j): j \in \mathbb{A}\}$ and $L=\sum_{j \in \mathbb{A}} d(j, \pi(j))$, where $\pi(j)$ denotes the preclustering assignment. Consider the weighted $k$-median problem on $\mathbb{M}$ where the weight of $m \in \mathbb{M}$ is defined to be the number of points that are assigned to $m$ in the preclustering, that is, $|\{j \mid j \in \mathbb{A}, \pi(j)=m\}|$. Then

(i) There exists a weighted $k$-median solution $\operatorname{sol}(\mathbb{M}, k)$ such that $C_{\text {sol }}(\mathbb{M}, k) \leq 2\left(L+C_{\text {opt }}(\mathbb{A}, k)\right)$.

(ii) Given any weighted $k$-median solution $\operatorname{sol}(\mathbb{M}, k)$, there exists a $k$-median solution $\operatorname{sol}(\mathbb{A}, k)$ such that $C_{\text {sol }}(\mathbb{A}, k) \leq \operatorname{sol}(\mathbb{M}, k)+L$. Consequently, there exists a $k$-median solution $\operatorname{sol}(\mathbb{A}, k)$ such that $C_{\text {sol }}(\mathbb{A}, k) \leq 2 \gamma\left(L+C_{\text {opt }}(\mathbb{A}, k)\right)+L$ and centers are restricted to $\mathbb{M}$, where $\gamma$ is the best approximation ratio for the $k$-median problem.

COROLlary 2.2. The result in Theorem 2.1 extends to

(i) the $k$-center problem;

(ii) the $k$-means problem with weaker constants, using a relaxed triangle inequality;

(iii) the $(k, t)$-median/means/center approximation on the weighted point set $\mathbb{M}$ (with $\gamma$ being the corresponding bicriteria approximation ratio), provided the preclustering does not ignore any points. Otherwise the total number of ignored points is the sum of the ignored points in the clustering and preclustering phases.

\section{3 ( $k, t)$-MEDIAN AND $(k, t)$-MEANS}

Our algorithm for distributed $(k, t)$-median clustering is provided in Algorithm 1. For integer pairs $(i, q)$, we consider the lexicographical order as partial order, that is,

$$
\left(i_{1}, q_{1}\right)<\left(i_{2}, q_{2}\right) \quad \text { if } \quad\left\{\begin{array}{l}
i_{1}<i_{2} ; \text { or } \\
i_{1}=i_{2} \text { and } q_{1}<q_{2} .
\end{array}\right.
$$

REMARK 1. In Line 17 of Algorithm 1, (i) no input point is ignored in the preclustering; (ii) if the preclustering aggregated $q$ points but the coordinator's algorithm chooses less than q copies (to exclude exactly t) then the proofs are not affected in any way.

We begin with a theorem about approximating $(k, t)$-median or means with a different trade-off from that in [4].

Theorem 3.1 (Proof Omitted). Let $\epsilon>0$. We can compute $\operatorname{sol}(Z, k,(1+\epsilon) t)$ and $\operatorname{sol}(Z,(1+\epsilon) k, t)$ for the $(k, t)$-median problem in $\tilde{O}\left(|Z|^{2}\right)$ time such that

$$
\begin{gathered}
C_{\mathrm{sol}}(Z, k,(1+\epsilon) t) \leq \max \{6,6 / \epsilon\} \cdot C_{\mathrm{opt}}(Z, k, t), \text { and } \\
C_{\mathrm{sol}}(Z,(1+\epsilon) k, t) \leq \max \{6,6 / \epsilon\} \cdot C_{\mathrm{opt}}(Z, k, t) .
\end{gathered}
$$

The result extends to the $(k, t)$-means problem with a slightly larger constant.

Throughout the rest of the section, we denote by $t_{i}^{*}$ the number of ignored points from $\mathbb{A}_{i}$ in the global optimum solution opt $(\mathbb{A}, k, t)$. We need the following lemmas.

\footnotetext{
${ }^{4}$ Stably means that when $\ell\left(i_{1}, q_{1}\right)=\ell\left(i_{2}, q_{2}\right)$, the sorting algorithm puts $\ell\left(i_{1}, q_{1}\right)$ before $\ell\left(i_{2}, q_{2}\right)$ if $\left(i_{1}, q_{1}\right)<\left(i_{2}, q_{2}\right)$ as defined in (4).

${ }^{4}$ Element of rank $r$ means the $r$-th element in a sorted list
}

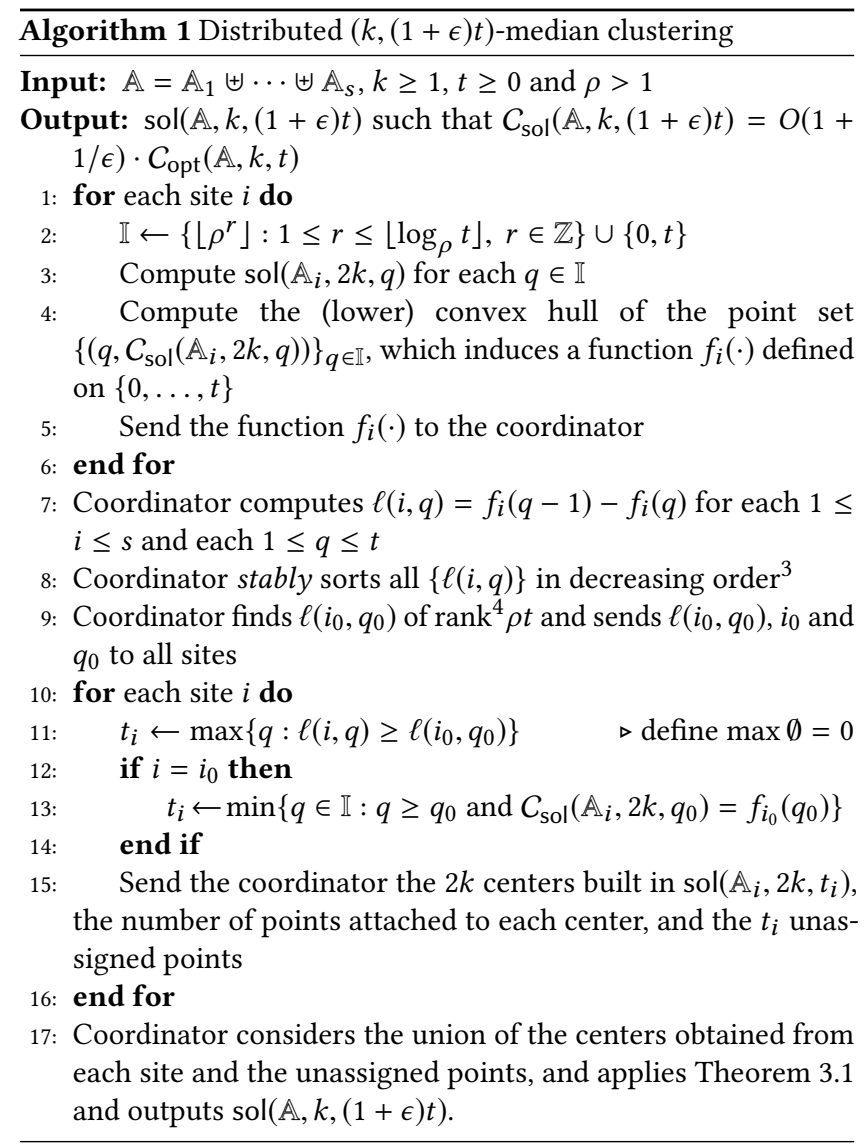

Lemma 3.2. It holds that $\sum_{i} C_{\text {opt }}\left(\mathbb{A}_{i}, k, t_{i}^{*}\right) \leq 2 C_{\text {opt }}(\mathbb{A}, k, t)$. For $(k, t)$-means the constant changes from 2 to 4 .

Proof. We shall use an argument used in [14]. Let $\pi_{\text {opt }}$ be the center projection function and $K$ be the set of optimum centers in the optimal solution opt $(\mathbb{A}, k, t)$. For each $\mathbb{A}_{i}$, we construct a solution $\operatorname{sol}\left(\mathbb{A}_{i}, k, t_{i}^{*}\right)$ by excluding the points excluded in $\operatorname{opt}(\mathbb{A}, k, t)$ and choosing $\left\{\arg \min _{u \in \mathbb{A}_{i}} d(u, k): k \in K\right\}$ to be the centers. Then

$$
C_{\text {sol }}\left(\mathbb{A}_{i}, k, t_{i}^{*}\right) \leq 2 \sum_{x \in \mathbb{A}_{i}} d\left(x, \pi_{\text {opt }}(x)\right) .
$$

Summing over $i$ yields $\sum C_{\text {sol }}\left(\mathbb{A}_{i}, k, t_{i}^{*}\right) \leq 2 C_{\text {opt }}(\mathbb{A}, k, t)$. The result for $k$-means follows from applying triangle inequality with $(a+$ $b)^{2} \leq 2\left(a^{2}+b^{2}\right)$.

Lemma 3.3. The $t_{1}, \ldots, t_{s}$ computed in Step 11 of Algorithm 1 minimizes $\sum_{i} f_{i}\left(t_{i}\right)$ subject to $\sum_{i} t_{i} \leq \rho t$ and $0 \leq t_{i} \leq t$.

Proof. Suppose that $t_{1}^{\prime}, \ldots, t_{s}^{\prime}$ is a minimizer. Since $f_{i}(\cdot)$ is nonincreasing for all $i$, it must hold that $\sum_{i} t_{i}^{\prime}=\rho t$. By the definition of $t_{i}$, it also holds that $\sum_{i} t_{i}=\rho t$. If $\left(t_{1}^{\prime}, \ldots, t_{s}^{\prime}\right) \neq\left(t_{1}, \ldots, t_{s}\right)$, there must exist $i, j$ such that $t_{i}^{\prime}>t_{i}$ and $t_{j}^{\prime}<t_{j}$. By the definition of $t_{i}$ and the sorting of $\{\ell(i, q)\}$, we know that

$$
\ell\left(i, t_{i}+1\right) \leq \ell\left(i_{0}, q_{0}\right), \quad \ell\left(j, t_{j}\right) \geq \ell\left(i_{0}, q_{0}\right) .
$$


From convexity of $f_{i}$ and that $t_{i}^{\prime} \geq t_{i}+1$ and $t_{j}^{\prime}+1 \leq t_{j}$, it follows that

$$
f_{i}\left(t_{i}^{\prime}-1\right)-f_{i}\left(t_{i}^{\prime}\right) \leq \ell\left(i_{0}, q_{0}\right) \leq f_{j}\left(t_{j}^{\prime}\right)-f_{j}\left(t_{j}^{\prime}+1\right)
$$

which means that increasing $t_{j}^{\prime}$ by 1 and decreasing $t_{i}^{\prime}$ by 1 will not decrease the sum

$$
G\left(q_{1}^{\prime}, \ldots, q_{s}^{\prime}\right):=\sum_{i}\left(f_{i}(0)-f_{i}\left(t_{i}^{\prime}\right)\right) .
$$

Therefore $\sum_{i} f_{i}\left(t_{i}^{\prime}\right)=\sum_{i} f_{i}(0)-G\left(t_{1}^{\prime}, \ldots, t_{s}^{\prime}\right)$ will not increase. We can continue this procedure until $\left(t_{1}^{\prime}, \ldots, t_{s}^{\prime}\right)=\left(t_{1}, \ldots, t_{s}\right)$.

LeMma 3.4. It holds for all $i \neq i_{0}$ that $t_{i} \in \mathbb{I}$ and $C_{\text {sol }}\left(\mathbb{A}_{i}, 2 k, t_{i}\right)=$ $f_{i}\left(t_{i}\right)$, where $i_{0}$ is computed in Step 9 and $t_{i}$ 's in Step 11 of Algorithm 1.

Proof. Since $0 \in \mathbb{I}$, we need only to consider the $i$ 's with $t_{i} \neq 0$. By the selection of $i_{0}$ and $q_{0}$, it must hold that

$$
\begin{array}{ll}
\ell\left(i, t_{i}\right) \geq \ell\left(i_{0}, q_{0}\right)>\ell\left(i, t_{i}+1\right) & \text { for } \quad i<i_{0} \\
\ell\left(i, t_{i}\right)>\ell\left(i_{0}, q_{0}\right) \geq \ell\left(i, t_{i}+1\right) & \text { for } \quad i>i_{0},
\end{array}
$$

which implies that $\ell\left(i, t_{i}\right)>\ell\left(i, t_{i}+1\right)$ whenever $i \neq i_{0}$, i.e.,

$$
f_{i}\left(t_{i}-1\right)-f_{i}\left(t_{i}\right)>f_{i}\left(t_{i}\right)-f_{i}\left(t_{i}+1\right), \quad i \neq i_{0} .
$$

Hence $\left(i, f_{i}\left(t_{i}\right)\right)$ is a vertex of the convex hull for all $i \neq i_{0}$, that is, $t_{i} \in \mathbb{I}$ and $f_{i}\left(t_{i}\right)=C_{\text {sol }}\left(\mathbb{A}_{i}, 2 k, t_{i}\right)$.

Now we are ready to bound the 'goodness' of local solutions.

Lemma 3.5. Let $\rho=2$. It holds that $\sum_{i} C_{\text {sol }}\left(\mathbb{A}_{i}, 2 k, t_{i}\right) \leq 12$. $C_{\text {opt }}(\mathbb{A}, 2 k, t)$ and $\sum_{i} t_{i} \leq 3 t$, where $t_{1}, \ldots, t_{s}$ are computed in Step 11 and may be updated in Step 15 of Algorithm 1.

Proof. Let $\hat{t}_{i}=\min \left\{q \in \mathbb{I}: q \geq t_{i}^{*}\right\}$. It follows from Lemma 3.2 with $\sum_{i} t_{i}^{*} \leq t$ that

$$
\begin{aligned}
2 C_{\mathrm{opt}}(\mathbb{A}, k, t) \geq \sum_{i} C_{\mathrm{opt}}\left(\mathbb{A}_{i}, k, t_{i}^{*}\right) \geq & \sum_{i} C_{\mathrm{opt}}\left(\mathbb{A}_{i}, k, \hat{t}_{i}\right) \\
& \geq \frac{1}{6} \sum_{i} C_{\mathrm{sol}}\left(\mathbb{A}_{i}, 2 k, \hat{t}_{i}\right),
\end{aligned}
$$

where the last inequality follows from Theorem 3.1 (applied with $\epsilon=\rho-1=1)$. Observe that $\hat{t}_{i} \leq 2 t_{i}^{*}$ and thus $\sum_{i} \hat{t}_{i} \leq 2 \sum_{i} t_{i}^{*} \leq 2 t$, and

$$
\sum_{i} C_{\text {sol }}\left(\mathbb{A}_{i}, 2 k, \hat{t}_{i}\right) \geq \sum_{i} f_{i}\left(\hat{t}_{i}\right) \geq \sum_{i} f_{i}\left(t_{i}\right),
$$

where the last equality follows from Lemma 3.3, and $t_{i}$ 's are computed in Step 11.

Now, by Lemma $3.4, f_{i}\left(t_{i}\right)=C_{\text {sol }}\left(\mathbb{A}_{i}, 2 k, t_{i}\right)$ for all except one $i$. The exceptional $t_{i}$ will be replaced by a bigger value, which will not increase $f_{i}\left(t_{i}\right)$ by the monotonicity of $f_{i}$, and the first part follows. This update will increase $\sum_{i} t_{i}$ by at most $t$ and thus $\sum_{i} t_{i} \leq 3 t$.

Lemma 3.5 and Theorem 3.1 together give the following. Note that $|\mathbb{I}|=O(\log t)$

Theorem 3.6. For the distributed $(k, t)$-median problem, Algorithm 1 with $\rho=2$ outputs $\operatorname{sol}(\mathbb{A}, k,(1+\epsilon) t)$ satisfying $C_{\text {sol }}(\mathbb{A}, k,(1+$ $\epsilon) t)) \leq O(1+1 / \epsilon) \cdot C_{\text {opt }}(\mathbb{A}, k, t)$. The sites communicate a total of $\tilde{O}(s k+t)$ bits of information with the coordinator over 2 rounds. The runtime at each site is $\tilde{O}\left(n_{i}^{2}\right)$ and the runtime at the coordinator is $\tilde{O}\left((s k+t)^{2}\right)$. The same result holds for $(k, t)$-means with larger constants in the approximation ratio and the runtime.
Proof. The communication cost is straightforward. By Lemma 3.5, the coordinator will solve the problem of at most $2 s k+3 t$ points. The claims on approximation ratio and the runtime then follow from Theorem 3.1, noting that it takes time $O(\mathbb{I} \log \mathbb{I})=\tilde{O}(1)$ to find the convex hull.

If we were only interested in the clustering and not the list of ignored points, we could set $\rho=1+\delta$ and change line 12 to line 15 of Algorithm 1 to the following. The sites do not send the ignored nodes but just the number of them, and the exceptional site runs a slightly more convoluted algorithm.

\section{2: if $i \neq i_{0}$ then}

13: $\quad$ Send the coordinator $t_{i}$, the $2 k$ centers built in $\operatorname{sol}\left(\mathbb{A}_{i}, 2 k, t_{i}\right)$ and the number of points attached to each center

14: else

15: $\quad t_{i, 1}=\max \left\{q \in \mathbb{I}: q \leq t_{i}\right.$ and $\left.C_{\mathrm{sol}}\left(\mathbb{A}_{i}, 2 k, q\right)=f_{i}(q)\right\}$

16: $\quad t_{i, 2}=\min \left\{q \in \mathbb{I}: q \geq t_{i}\right.$ and $\left.C_{\mathrm{sol}}\left(\mathbb{A}_{i}, 2 k, q\right)=f_{i}(q)\right\}$

17: $\quad$ Combine $\operatorname{sol}\left(\mathbb{A}_{i}, 2 k, t_{i, 1}\right)$ and $\operatorname{sol}\left(\mathbb{A}_{i}, 2 k, t_{i, 2}\right)$ to form a solution $\operatorname{sol}\left(\mathbb{A}_{i}, 4 k, t_{i}\right)$ by taking the union of the medians, attaching each point to the closest center among the combined centers, and ignoring the points with largest $t_{i}$ distances.

18: Send to the coordinator $t_{i}$, the combined centers and the number of points attached to each center.

19: end if

Observe that Lemma 3.5 still holds with $\sum_{i} t_{i} \leq(1+\delta) t$, since we are not changing the exceptional $t_{i}$. For the exceptional site $i$, suppose that $t_{i}=(1-\theta) t_{i, 1}+\theta t_{i, 2}$ for some $\theta \in(0,1)$, we have $(1-\theta) f_{i}\left(t_{i, 1}\right)+\theta f_{i}\left(t_{i, 2}\right) \leq f_{i}\left(t_{i}\right)$. We now argue the next critical lemma.

Lemma 3.7. $C_{\text {sol }}\left(\mathbb{A}_{i}, 4 k, t_{i}\right) \leq(1-\theta) f_{i}\left(t_{i, 1}\right)+\theta f_{i}\left(t_{i, 2}\right)$.

Proof. We will prove the lemma by carefully designing an assignment of $n-t_{i}$ points to the $4 k$ centers which is bounded above by the right hand side. Since choosing the minimum $n-t_{i}$ distances will only result in a smaller value, the lemma would follow.

For $j=1,2$, let $\pi_{j}$ be the center projection function in $\operatorname{sol}\left(\mathbb{A}_{i}, 2 k, t_{i, j}\right)$ and $P_{i}$ the set of clustered points in $\operatorname{sol}\left(\mathbb{A}_{i}, 2 k, t_{i, j}\right)$. For $x \in P_{1} \cap P_{2}$, we attach $x$ to the nearer one between the two centers $\pi_{1}(x)$ and $\pi_{2}(x)$, and the incurred cost is

$$
\min \left\{d\left(x, \pi_{1}(x)\right), d\left(x, \pi_{2}(x)\right)\right\} \leq(1-\theta) d\left(x, \pi_{1}(x)\right)+\theta d\left(x, \pi_{2}(x)\right) .
$$

For $x \in P_{1} \triangle P_{2}$, since only one of $\pi_{1}(x)$ and $\pi_{2}(x)$ exist, we abbreviate it as $\pi(x)$ for simplicity. Define $h(x)$ for each $x \in P_{1} \triangle P_{2}$ as

$$
h(x)= \begin{cases}(1-\theta) \cdot d(x, \pi(x)), & x \in P_{1} \backslash P_{2} ; \\ \theta \cdot d(x, \pi(x)), & x \in P_{2} \backslash P_{1} .\end{cases}
$$

Let $r=\left|P_{1} \cap P_{2}\right|, r_{1}=\left|P_{1} \backslash P_{2}\right|$ and $r_{2}=\left|P_{2} \backslash P_{1}\right|$. It holds that $r+r_{1}=n-t_{i, 1}$ and $r+r_{2}=n-t_{i, 2}$, thus $r_{1}>r_{2}$ and

$$
(1-\theta) r_{1}+\theta r_{2}=n-t_{i}-r \text {. }
$$

Define $Q_{1}=P_{1} \backslash P_{2}$ and $Q_{2}=P_{2} \backslash P_{1}$. Pick $x=\arg \min _{z \in Q_{1} \cup Q_{2}} h(z)$. If $x \in Q_{1}$, pick an arbitrary $u \in Q_{2}$, otherwise pick $u \in Q_{1}$. Attach $x$ to $\pi(x)$ in the $4 k$-center solution we are constructing and mark $u$ 
as outlier. Note that this incurs a cost of

$$
d(x, \pi(x)) \leq \begin{cases}(1-\theta) d(x, \pi(x))+\theta d(u, \pi(u)), & x \in Q_{1} ; \\ (1-\theta) d(u, \pi(u))+\theta d(x, \pi(x)), & x \in Q_{2},\end{cases}
$$

by our choice of $x$, because one of the combination terms is exactly $h(x)$ and it is smaller than $h(u)$, which is exactly the other term. Then we remove $x$ and $u$ from $Q_{1}$ or $Q_{2}$ depending on the case. Now, $\left|Q_{1}\right|=r_{1}-1$ and $\left|Q_{2}\right|=r_{2}-1$, and note that

$$
(1-\theta)\left(r_{1}-1\right)+\theta\left(r_{2}-1\right)=n-t_{i}-r-1 .
$$

Since $r_{1}>r_{2}$, we can continue this process until $Q_{2}=\emptyset$. At this point we have run the procedure above $r_{2}$ times, and it holds that

$$
(1-\theta) r_{1}=n-t_{i}-r-r_{2} .
$$

Note that $r_{1} \geq n-t_{i}-r-r_{2}$, so we can choose $E \subseteq Q_{1}$ to be the points with smallest $n-t_{i}-r-r_{2}$ values of $h$. Attach points in $E$ to their respective centers and mark the remaining points in $Q_{1}$ as outliers. This incurs a cost of

$$
\begin{aligned}
\sum_{x \in E} d(x, \pi(x)) \leq \frac{n-t_{i}-r-r_{2}}{r_{1}} & \sum_{x \in Q_{1}} d(x, \pi(x)) \\
& =(1-\theta) \sum_{x \in Q_{1}} d(x, \pi(x))
\end{aligned}
$$

In total we have assigned $r+r_{2}+\left(n-t_{i}-r-r_{2}\right)=n-t_{i}$ points as desired. The desired upper bound on cost follows from (i) summing both sides of (5) over $P_{1} \cap P_{2}$; (ii) summing both sides of (6) over $x$ and the corresponding $u$ during the pairing procedure; and (iii) Equation (7). Note that (ii) covers $\left(P_{1} \triangle P_{2}\right) \backslash Q_{1}$, where $Q_{1}$ is the post-pairing set.

As a consequence of Lemma 3.7, $C_{\text {sol }}\left(\mathbb{A}_{i}, 4 k, t_{i}\right) \leq f_{i}\left(t_{i}\right)$. Thus the upper bound on the approximation ratio still holds. Finally, note that $|\mathbb{I}|=\tilde{O}(1 / \delta)$ and we conclude that

Theorem 3.8. For the distributed $(k, t)$-median problem, the modified Algorithm 1 with $\rho=1+\delta$ outputs $\operatorname{sol}(\mathbb{A}, k,(2+\epsilon+\delta) t)$ satisfying $C_{\text {sol }}(\mathbb{A}, k,(2+\epsilon+\delta) t) \leq O(1+1 / \epsilon) \cdot C_{\text {opt }}(\mathbb{A}, k, t)$. The sites communicate a total of $\tilde{O}\left(s \delta^{-1}+s k B\right)$ bits of information with the coordinator over 2 rounds. The runtime on site $i$ is $\tilde{O}\left(n_{i}^{2} / \delta\right)$ and the runtime on the coordinator is $\tilde{O}\left((s k)^{2}\right)$. The same result holds for $(k, t)$-means with a larger constant in the approximation ratio.

\subsection{Subquadratic-time Centralized Algorithm}

We now show an unusual application of Theorem 3.6 in speeding up existing constant-factor approximation algorithms for $(k, t)$-median (or means). Note that the centralized bicriteria approximation algorithms in Charikar [4] are $\tilde{O}\left(n^{3}\right)$ from $n$ points, and while the modifications in Theorem 3.1 improve the running time to $\tilde{O}\left(n^{2}\right)$, this leaves open the important question: Are there algorithms with provable constant factor approximation guarantees which are subquadratic? Observe that the question is even more pertinent in the context of unicriterion approximation, for which the only known result is a $\tilde{O}\left(n^{3} k^{2} t^{2}\right)$-time constant-factor approximation of $(k, t)$ median [6]. In the sequel we show that the running time can be brought to almost linear time. The improvement arises from the fact that we can simulate a distributed algorithm sequentially.
Lemma 3.9. Suppose that we are given a $\tilde{O}\left(n^{1+\alpha_{0}} k^{2}\right)$ time algorithm for bicriteria approximation which produces $2 k$ centers or $2 t$ outliers with approximation factor $\gamma$, where $\alpha_{0} \leq 1$. Then we can produce a similar algorithm with running time $\tilde{O}\left(t^{2}\right)+\tilde{O}\left(n^{\frac{2+2 \alpha_{0}}{2+\alpha_{0}}} k^{2}\right)$ and approximation $c_{0} \gamma$ for some absolute constant $c_{0}>0$.

Proof. We will apply Theorem 3.6 after dividing the data arbitrarily in $s$ pieces of size $n / s$. The sequential simulation of the $s$ sites will take time $\tilde{O}\left(s(n / s)^{1+\alpha_{0}} k^{2}\right)$ based on the statement of the lemma. The coordinator will require time $\tilde{O}\left((s k+t)^{2}\right)=\tilde{O}\left(s^{2} k^{2}\right)+$ $\tilde{O}\left(t^{2}\right)$. Observe that we can now balance $n^{1+\alpha_{0}}=s^{2+\alpha_{0}}$, which provides us the optimum $s$ to use and achieve a running time of

$$
\tilde{O}\left(t^{2}\right)+\tilde{O}\left(s^{2} k^{2}\right)=\tilde{O}\left(t^{2}\right)+\tilde{O}\left(n^{\frac{2+2 \alpha_{0}}{2+\alpha_{0}}} k^{2}\right) .
$$

Theorem 3.10. Let $\alpha>0$ and suppose that $t \leq \sqrt{n}$. There exists a centralized algorithm for the $(k, t)$-median problem that runs in $\tilde{O}\left(n^{1+\alpha} k^{2}\right)$ time and outputs a solution $\operatorname{sol}(\mathbb{A}, k, 2 t)$ satisfying $C_{\text {sol }}(\mathbb{A}, k, 2 t) \leq(1+1 / \alpha)^{O(1)} C_{\text {opt }}(\mathbb{A}, k, t)$.

Proof. Note that the algorithm in Theorem 3.1 has runtime $\tilde{O}\left(n^{2}\right)$, so we can take $\alpha_{0}=1$ in Lemma 3.9 to obtain an algorithm of approximation ratio $\gamma=6$ and runtime $\tilde{O}\left(t^{2}+n^{4 / 3} k^{2}\right)$, which is $\tilde{O}\left(n^{4 / 3} k^{2}\right)$ by our assumption that $t \leq \sqrt{n}$. Repeatedly applying Lemma 3.9 for $j$ times gives an algorithm of runtime $\tilde{O}\left(n^{1+1 /\left(2^{j}-1\right)} k^{2}\right)$ and approximation ratio $\left(c_{0} \gamma\right)^{j}$. Let $j=\log (1+$ $1 / \alpha)$, the runtime becomes $O\left(n^{1+\alpha} k^{2}\right)$ and the approximation ratio $(1+1 / \alpha)^{\log \left(c_{0} \gamma\right)}=(1+1 / \alpha)^{O(1)}$.

REMARK 2. We remark that

(i) the theorem above also holds for $\operatorname{sol}(\mathbb{A}, 2 k, t)$, where the number of centers, instead of the outliers, is relaxed.

(ii) for the unicriterion approximation, if we use the algorithm of runtime $\tilde{O}\left(n^{3} t^{2} k^{2}\right)$ from [6] instead of the result of Theorem 3.1, we need to balance $s^{3}$ and $s(n / s)^{1+\alpha_{0}}$ for an analogy of Lemma 3.9, which will eventually lead to an algorithm of runtime $O\left(n^{1+\alpha} t^{2} k^{2}\right)$, provided that $t \leq n^{1 / 5}$.

\section{$4(k, t)$-CENTER CLUSTERING}

Our algorithm for $(k, t)$-center clustering is presented in Algorithm 2. It is similar to Algorithm 1 but only simpler, because the preclustering stage admits a simpler algorithm due to Gonzalez [13]. For the $k$-center problem on a point set $Z$ of $n$ points, Gonzalez's algorithm outputs a re-ordering of points in $Z$, say, $p_{1}, \ldots, p_{n}$, such that for each $1 \leq r \leq n$, the solution $\operatorname{sol}(Z, r)$ of choosing $\left\{p_{1}, \ldots, p_{r}\right\}$ as the $r$ centers is a 2-approximation for the $r$-center problem on $Z$, i.e., $C_{\text {sol }}(Z, r) \leq 2 C_{\text {opt }}(Z, r)$.

The core argument is that the $k$-center algorithm of Gonzalez can be used to simultaneously (a) precluster the local data into local solutions and (b) provide a witness that can be compared globally.

Remark 3. In Algorithm 2, (i) none of the original points is ignored in the preclustering, and (ii) it is possible that the preclustering aggregated $q$ points but the coordinator's algorithm chooses less than $q$ copies to exclude exactly $t$ points. This does not affect the proofs of $(k, t)$-center clustering. 


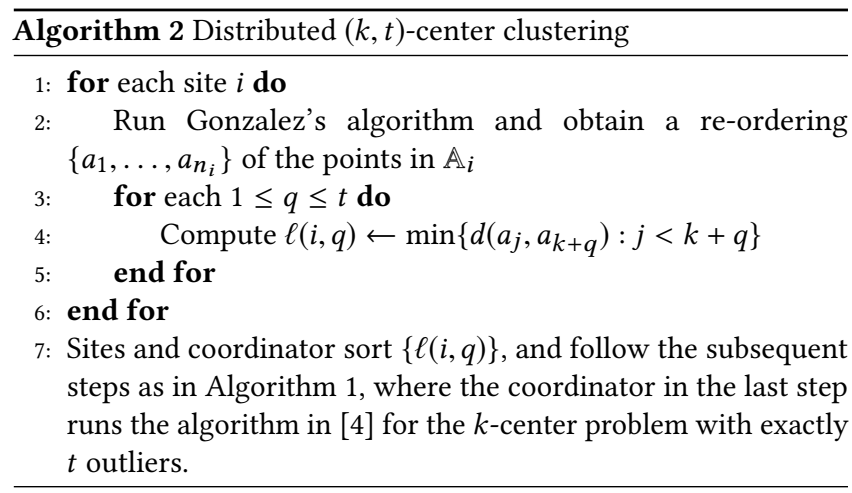

We now analyze the performance of Algorithm 2. Denote by $t_{i}^{*}$ the number of points ignored from $\mathbb{A}_{i}$ in the global optimum solution opt $(\mathbb{A}, k, t)$. First we show two structural lemmas.

LemmA 4.1. $2 C_{\text {opt }}\left(\mathbb{A}_{i}, k, t\right) \geq \max _{i} C_{\text {opt }}\left(\mathbb{A}_{i}, k, t_{i}^{*}\right)$.

Proof. Use the same argument in the proof of Lemma 3.2.

LEMmA 4.2. $\max _{i} C_{\text {opt }}\left(\mathbb{A}_{i}, k, t_{i}^{*}\right) \geq \min _{\sum_{i} t_{i} \geq t}\left(\max _{i} C_{\text {opt }}\left(\mathbb{A}_{i}, k, t_{i}\right)\right)$.

Proof. It follows from the fact that $\sum_{i} t_{i}^{*}=t$.

THeOREM 4.3. For the distributed $(k, t)$-center problem, Algorithm 2 outputs $\operatorname{sol}(\mathbb{A}, k, t)$ satisfying $C_{\mathrm{sol}}(\mathbb{A}, k, t) \leq O(1) \cdot C_{\text {opt }}(\mathbb{A}, k, t)$. The sites communicate a total of $\tilde{O}((s k+t) B)$ bits of information to the coordinator over 2 rounds. The runtime on site i is $\tilde{O}\left((k+t) n_{i}\right)$ and the runtime on the coordinator is $\tilde{O}\left((s k+t)^{2}\right)$.

Proof. The approximation ratio follows from a similar argument to that of Theorem 3.6, using Lemma 4.1 and 4.2. The coordinator runtime follows from [4, Theorem 3.1] and the site runtime from [13], noting that we need only the first $k+t$ points of the reordering of each $\mathbb{A}_{i}$. The communication cost is clear from Algorithm 2 .

\section{CLUSTERING UNCERTAIN INPUT}

Recall that in the setting of clustering with uncertainty there is an underlying metric space $(\mathcal{P}, d)$. We are given a set of input nodes $j \in \mathbb{A}$ which correspond to distributions $\mathcal{D}_{j}$ on $\mathcal{P}$. In this section we shall use nodes to indicate the input and points to indicate deterministic objects in the metric space $\mathcal{P}$. We shall denote by $\sigma(j)$ a realization of node $j$ and by $\pi(j)$ the center node to which $j$ is attached. Our goal in the $(k, t)$-median problem in this context is to compute

$$
\min _{\substack{K \subseteq \mathcal{P}, \mathbb{Q} \\|K| \leq k,|\mathbb{A}| \leq t}}\left[\sum_{j \in \mathbb{A} \mid \mathbb{O}}\left(\min _{\pi(j)} \underset{\sigma}{\mathbb{E}}[d(\sigma(j), \pi(j))]\right)\right] .
$$

For $(k, t)$-means we use $d^{2}(\cdot, \cdot)$ and for $(k, t)$-center-pp we use $\max _{j}$ instead of $\sum_{j}$.

Define $\widehat{d}: \mathbb{A} \times \mathcal{P} \rightarrow \mathbb{R}$ as $\widehat{d}(j, p)=\mathbb{E}_{\sigma}[d(\sigma(j), p)]$, the objective function (8) is then reduced to the usual $(k, t)$-median problem with the new distance function $\widehat{d}$. However, this definition only allows the computation of distance between an input node and a point in $\mathcal{P}$. To extend $\widehat{d}$ to a pair of input nodes, the site holding $\mathbb{A}_{i}$ will need to know the point set $\bigcup_{j \in \mathbb{A}_{i^{\prime}}} \operatorname{supp}\left(\mathcal{D}_{j}\right)$ from some other site $i^{\prime}$. This will blow up the communication cost, and thus naively using this distance function in combination with the algorithms developed previously will not work well. To circumvent this issue we combine the notion of 1-median introduced in [8] along with the framework in Theorem 2.1, and introduce a compression scheme to evaluate distances.

Definition 5.1. For each node $j$, define its 1-median and 1-mean to be

$$
y_{j}=\underset{y \in \mathcal{P}}{\arg \min } \underset{\sigma}{\mathbb{E}}[d(\sigma(j), y)], \quad y_{j}^{\prime}=\underset{y \in \mathcal{P}}{\arg \min } \underset{\sigma}{\mathbb{E}}\left[d^{2}(\sigma(j), y)\right],
$$

respectively.

Definition 5.2 (Compressed graph). The compressed graph $G(\mathbb{A})$ is a weighted graph on vertices $\mathcal{P} \cup\left\{p_{j}\right\}_{j \in \mathbb{A}}$, where the edges are as follows: (1) each pair $(u, v) \in \mathcal{P}$ is an edge with weight $d(u, v)$, and (2) for each $j \in \mathbb{A}$, the vertex $p_{j}$ is connected only to $y_{j}$ with weight $\ell_{j}=\mathbb{E}_{\sigma}\left[d\left(\sigma(j), y_{j}\right)\right]$. Define the distance $d_{G}(u, v)$ between two vertices $u, v$ in $G$ to be the length of the shortest path between $u$ and $v$ in $G$.

For the compressed graph $G$, we can also consider the following $(k, t)$-median problem, where we restrict the demand points to $\left\{p_{j}\right\}$ and the possible centers to $\left\{y_{j}\right\}$, and the distance function is the length of shortest path on $G$. We continue to use the notations $\operatorname{sol}(G, k, t), C_{\mathrm{sol}}(G, k, t)$, etc., to denote the solution and the corresponding cost of $(k, t)$-median problem on $G$. The following two lemmas show that $(k, t)$-median problem in Eqn (8) is, up to some constant factor in the approximation ratio, equivalent to the $(k, t)$-median problem on the compressed graph.

Lemma 5.3. If there exists a solution $\operatorname{sol}(\mathbb{A}, k, t)$ of $\operatorname{cost} C_{\text {sol }}(\mathbb{A}, k, t)$ to the objective in Equation (8), then there exists a solution $\operatorname{sol}(G(\mathbb{A}), k, t)$ on the compressed graph such that $C_{\mathrm{sol}}(G(\mathbb{A}), k, t) \leq 5 C_{\text {sol }}(\mathbb{A}, k, t)$.

Proof. Let $\mathbb{A}^{\prime}$ be the set of clustered nodes in the feasible $(k, t)$ median solution of the original problem with the objective in (8). Define the set of center points $M=\left\{y_{j}: j \in \mathbb{A}^{\prime}\right\}$. For each $j \in \mathbb{A}^{\prime}$, let $y_{\pi(j)}=\arg \min _{y \in M} d(\pi(j), y)$. Let $\operatorname{sol}(G(\mathbb{A}), k, t)$ be the solution of connecting each point $p_{j}\left(j \in \mathbb{A}^{\prime}\right)$ to $y_{\pi(j)}$ in the compressed graph $G$. We try to upper bound the cost $C_{\text {sol }}(G(\mathbb{A}), k, t)$ :

$$
\begin{array}{ll}
C_{\text {sol }}(G(\mathbb{A}), k, t)=\sum_{j \in \mathbb{A}^{\prime}} d_{G}\left(y_{\pi(j)}, p_{j}\right) & \text { (definition of } C_{\text {sol }} \text { ) } \\
=\sum_{j \in \mathbb{A}^{\prime}}\left(d\left(y_{\pi(j)}, y_{j}\right)+d_{G}\left(y_{j}, p_{j}\right)\right) & \text { (definition of } d_{G} \text { ) } \\
\leq \sum_{j \in \mathbb{A}^{\prime}} d\left(y_{\pi(j)}, \pi(j)\right)+\sum_{j \in \mathbb{A}^{\prime}} d\left(\pi(j), y_{j}\right)+\sum_{j \in \mathbb{A}^{\prime}} d_{G}\left(y_{j}, p_{j}\right) \\
\leq 2 \sum_{j \in \mathbb{A}^{\prime}} d\left(\pi(j), y_{j}\right)+\sum_{j \in \mathbb{A}^{\prime}} \ell_{j},
\end{array}
$$

where the last line follows from $d\left(y_{\pi(j)}, \pi(j)\right) \leq d\left(\pi(j), y_{j}\right)$ by the definition (optimality) of $y_{\pi(j)}$.

Observe that for any realization $\sigma(j)$, it holds that

$$
d\left(y_{j}, \pi(j)\right) \leq d\left(y_{j}, \sigma(j)\right)+d(\sigma(j), \pi(j)) .
$$


Taking expectation over $\sigma$,

$$
d\left(y_{j}, \pi(j)\right) \leq \underset{\sigma}{\mathbb{E}} d\left(y_{j}, \sigma(j)\right)+\underset{\sigma}{\mathbb{E}} d(\sigma(j), \pi(j))=\ell_{j}+\underset{\sigma}{\mathbb{E}} d(\sigma(j), \pi(j)) .
$$

Summing over $j \in \mathbb{A}^{\prime}$,

$$
\sum_{j \in A^{\prime}} d\left(y_{j}, \pi(j)\right) \leq \sum_{j \in \mathbb{A}^{\prime}} \ell_{j}+\sum_{j \in \mathbb{A}^{\prime}} \underset{\sigma}{\mathbb{E}} d(\sigma(j), \pi(j)) \leq \sum_{j \in \mathbb{A}^{\prime}} \ell_{j}+C_{\text {opt }}(\mathbb{A}, k, t) .
$$

We next bound $\sum_{j \in \mathbb{A}^{\prime}} \ell_{j}$. This is exactly the cost of connecting each $j \in \mathbb{A}^{\prime}$ to its 1-median, which is the optimal solution of at most $n-t$ centers for $\mathbb{A}^{\prime}$. The optimal cost for $n-t$ centers is clearly less than that for $k$ centers and hence $\sum_{j \in \mathbb{A}^{\prime}} \ell_{j} \leq C_{\text {opt }}(\mathbb{A}, k, t)$.

Therefore $C_{\text {sol }}(G(\mathbb{A}), k, t) \leq 2 \cdot 2 C_{\text {opt }}(\mathbb{A}, k, t)+C_{\text {opt }}(\mathbb{A}, k, t)=$ $5 C_{\text {opt }}(\mathbb{A}, k, t)$ as claimed.

LEMMA 5.4. If there exists a solution $\operatorname{sol}(G(\mathbb{A}), k, t)$ of cost $C_{\text {sol }}(G(\mathbb{A}), k, t)$ on the compressed graph, then there exists a solution $\operatorname{sol}(\mathbb{A}, k, t)$ for the problem formulated in $(8)$ such that $C_{\mathrm{sol}}(\mathbb{A}, k, t) \leq$ $2 C_{\text {sol }}(G(\mathbb{A}), k, t)$.

Proof. Let $\mathbb{A}^{\prime \prime}$ be the set of clustered nodes in $\operatorname{sol}(G(\mathbb{A}), k, t)$. A similar argument of increasing the number of centers as in Lemma 5.3 yields that $\sum_{j \in \mathbb{A}^{\prime \prime}} \ell_{j} \leq C_{\text {sol }}(G(\mathbb{A}), k, t)$. Suppose that $p_{j}$ is assigned to $\pi(j)$ in $\operatorname{sol}(G(\mathbb{A}), k, t)$ in the compressed graph. Note that $\pi(j) \in \mathcal{P}$. Let $\operatorname{sol}(\mathbb{A}, k, t)$ be the solution of attaching $j$ to $\pi(j)$ in $\mathcal{P}$, and the cost can be bounded as

$$
\begin{aligned}
C_{\text {sol }}(\mathbb{A}, k, t) & \left.=\sum_{j \in \mathbb{A}^{\prime \prime}} \underset{\sigma}{\mathbb{E}}(d(\sigma(j), \pi(j))) \quad \text { (definition of } C_{\text {sol }}\right) \\
& \leq \sum_{j \in \mathbb{A}^{\prime \prime}} \underset{\sigma}{\mathbb{E}}\left(d\left(\sigma(j), y_{j}\right)\right)+\sum_{j \in \mathbb{A}^{\prime \prime}} d\left(y_{j}, \pi(j)\right) \\
& \leq \sum_{j \in \mathbb{A}^{\prime \prime}} \ell_{j}+\sum_{j \in \mathbb{A}^{\prime \prime}} d_{G}\left(p_{j}, \pi(j)\right) \\
& \leq 2 C_{\text {sol }}(G(\mathbb{A}), k, t), \quad \text { (definitiongle inequality) }
\end{aligned}
$$

where the third line follows from $d_{G}\left(p_{j}, \pi(j)\right)=d\left(p_{j}, y_{j}\right)+d\left(y_{j}, \pi(j)\right) \geq$ $d\left(y_{j}, \pi(j)\right)$.

The equivalence between the original problem and the one on the compressed graph also holds for the $(k, t)$-center-pp and the $(k, t)$-means problems.

LEMMA 5.5. Lemma 5.3 and Lemma 5.4 both hold

(a) for $(k, t)$-center-pp with the same constants; and

(b) for $(k, t)$-means with slightly larger constants.

Proof. (a) Observe that $\sum_{j}$ is replaced with $\max _{j}$ and Equation (9) rewrites to

$$
\max _{j \in \mathbb{A}^{\prime}} d\left(y_{j}, \pi(j)\right) \leq \max _{j \in \mathbb{A}^{\prime}} \ell_{j}+C_{\text {opt }}(\mathbb{A}, k, t) .
$$

The remainder of the equations hold with this transformation.

(b) Note that we used triangle inequality in the proof above. $\mathrm{Al}-$ though the square of the distance does not obey the triangle inequality, we can nevertheless apply $(a+b)^{2} \leq 2 a^{2}+2 b^{2}$ after the triangle inequality. The derivations above will go through and the results hold with slightly larger constants.

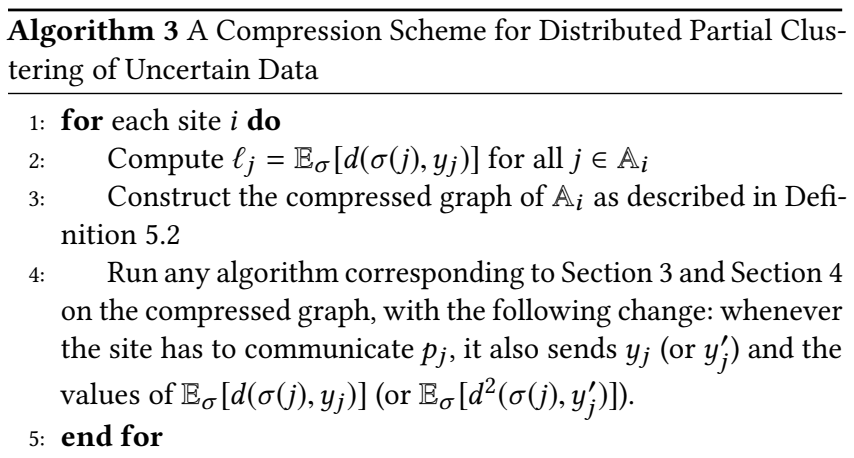

The overall algorithm is summarized in Algorithm 3. Note that we cannot just cluster the $\left\{y_{j}\right\}$; the graph is necessary. To implement the algorithm, we need to show that each site is able to compute the distance function individually. Indeed, note that any site that contains $p_{j}$ will also contain the corresponding $y_{j}$ or $y_{j}^{\prime}$ and the value $\mathbb{E}_{\sigma}\left[d\left(\sigma(j), y_{j}\right)\right]$ or $\mathbb{E}_{\sigma}\left[d^{2}\left(\sigma(j), y_{j}^{\prime}\right)\right]$ respectively. Therefore the distance oracle on the graph can be implemented by the site in constant time.

Theorem 5.6. For the distributed $(k, t)$-median problem, Algorithm 3 outputs $\operatorname{sol}(\mathbb{A}, k,(1+\epsilon) t)$ such that $C_{\text {sol }}(\mathbb{A}, k,(1+\epsilon) t)=$ $O(1+1 / \epsilon) \cdot C_{\text {opt }}(\mathbb{A}, k, t)$. The sites communicate a total of $\tilde{O}((s k+t) B)$ bits of information to the coordinator over 2 rounds. The runtime on site $i$ is $\tilde{O}\left(n_{i}^{2}+n_{i} T\right)$, where $T$ is the runtime to compute 1-median, and the runtime on the coordinator is $\tilde{O}\left((s k+t)^{2}\right)$. The same result holds for the $(k, t)$-median and center-pp problems with larger constants.

Proof. By Lemma 5.4 for the median problem and Lemma 5.5 for the means and center-pp problems, it suffices to show that we can solve the $(k, t)$-median problem on the compressed graph. The result then follows from Theorem 3.6 and Theorem 3.8 with the following amendments: When a site sends the $t$ or $t_{i}$ potential outliers, it needs to send the $y_{j}$ and the corresponding values $\mathbb{E}_{\sigma}\left[d\left(\sigma(j), y_{j}\right)\right]$ or $\mathbb{E}_{\sigma}\left[d^{2}\left(\sigma(j), y_{j}^{\prime}\right)\right]$, which at most doubles the communication cost. The runtime is increased by $O\left(n_{i} T\right)$ due to Step 2 since computing $\ell_{j}$ on the compressed graph takes $O(T)$ time.

Other results claimed in Table 2 follow from analogous amendments to Theorem 3.8.

The global $k$-Center case. We now focus on $(k, t)$-center-g. In this setting $\mathcal{D}_{j}$ 's are independent and we optimize

$$
\min _{\substack{K \subseteq \mathcal{P}, \mathbb{O} \subseteq \mathbb{A} \\|K| \leq k,|\mathbb{O}| \leq t}}\left(\underset{\sigma \sim \prod_{j}}{\mathbb{E}} \mathcal{D}_{j}\left[\max _{j \in \mathbb{A} \mid \mathbb{O}} d(\sigma(j), \pi(j))\right]\right) .
$$

Definition 5.7 (Truncated distance [15]). For $\tau \geq 0$, define $\mathcal{L}_{\tau}$ : $\mathcal{P} \times \mathcal{P} \rightarrow \mathbb{R}$ as $\mathcal{L}_{\tau}(u, v)=\max \{d(u, v)-\tau, 0\}$ and $\rho_{\tau}: \mathbb{A} \times \mathcal{P} \rightarrow \mathbb{R}$ as $\rho_{\tau}(j, u)=\mathbb{E}_{\sigma}\left[\mathcal{L}_{\tau}(\sigma(j), u)\right]$. Note that $\mathcal{L}_{\tau}(\cdot, \cdot)$ is not a metric for $\tau>0$.

Definition 5.8. Given a node set $Z \subseteq \mathbb{A}$, let $\mathcal{P}(Z) \subseteq \mathcal{P}$ be the associated point set corresponding to possible realizations of nodes in $Z$. Let $\operatorname{sol}\left(Z, k, t, \rho_{\tau}\right)$ and opt $\left(Z, k, t, \rho_{\tau}\right)$ be a solution by algorithm and the global optimum solution respectively to the $(k, t)$-median problem on node set $Z$ where the centers are restricted to $\mathcal{P}(Z)$ 
and the weighted assignment cost of assigning node $j \in Z$ to center $m \in \mathcal{P}(Z)$ is $\rho_{\tau}(j, m)$. The costs $C_{\text {sol }}\left(Z, k, t, \rho_{\tau}\right)$ and $C_{\text {opt }}\left(Z, k, t, \rho_{\tau}\right)$ are defined analogously.

Let $d_{\min }$ and $d_{\text {max }}$ denote the minimum and the maximum distance, respectively, between two distinct points in $\mathcal{P}$ and let $\Delta=$ $d_{\max } / d_{\min }$. The algorithm is presented in Algorithm 4.

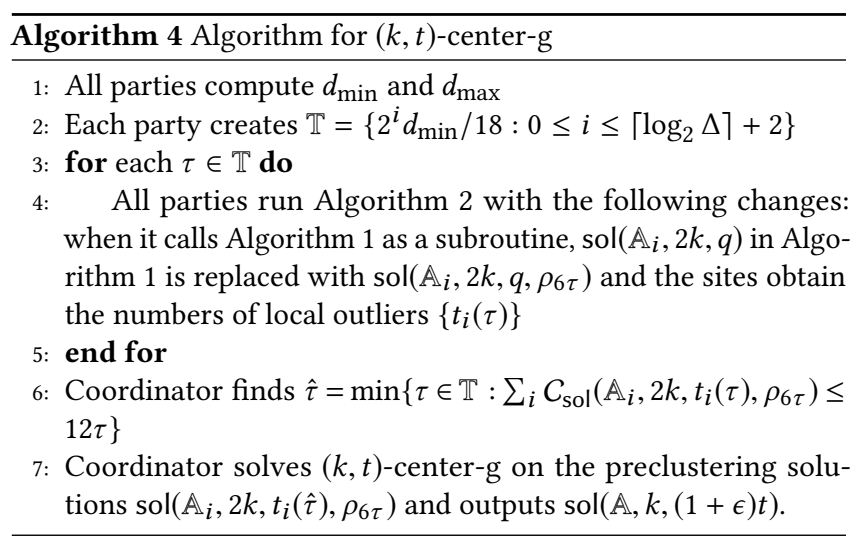

Now we try to analyze the performance of Algorithm 4. We first show an analogy of Theorem 3.1 that we can compute a constant approximation to $C_{\text {opt }}\left(Z, k, t, \rho_{\tau}\right)$. The proof is omitted.

Lemma 5.9. Let $\tau \geq 0$. For the $(k, t)$-center problem on $Z$, we can compute in $\tilde{O}((k+t)|Z|)$ time $\operatorname{sol}\left(Z, k,(1+\epsilon) t, \rho_{9 \tau}\right)$ or $\operatorname{sol}(Z,(1+$ $\left.\epsilon) k, t, \rho_{3 \tau}\right)$ such that

$$
\begin{aligned}
& C_{\text {sol }}\left(Z, k,(1+\epsilon) t, \rho_{9 \tau}\right) \leq \max \{6,6 / \epsilon\} \cdot C_{\mathrm{opt}}\left(Z, k, t, \rho_{\tau}\right) \\
& C_{\mathrm{sol}}\left(Z,(1+\epsilon) k, t, \rho_{3 \tau}\right) \leq \max \{6,6 / \epsilon\} \cdot C_{\mathrm{opt}}\left(Z, k, t, \rho_{\tau}\right)
\end{aligned}
$$

We next show that the $\hat{\tau}$ computed in Step 6 is a good choice of $\tau$ and will ensure that the preclustering solutions $\operatorname{sol}\left(\mathbb{A}_{i}, 2 k, t_{i}(\hat{\tau}), \rho_{2 \hat{\tau}}\right)$ can be combined to yield a good global solution. Specifically we have the following two lemmas.

Lemma 5.10. The $\hat{\tau}$ computed in Step 6 satisfies the following two conditions.

(i) $\sum_{i} C_{\text {sol }}\left(\mathbb{A}_{i}, 2 k, t_{i}(\hat{\tau}), \rho_{6}\right) \leq 12 \hat{\tau}$;

(ii) $\sum_{i} C_{\text {opt }}\left(\mathbb{A}_{i}, k, t_{i}^{\prime}, \rho_{2 \hat{\tau}}\right) \geq 2 \hat{\tau}$ for all $\left\{t_{i}^{\prime}\right\}$ s.t. $\sum_{i} t_{i}^{\prime} \leq t$,

Proof. Note that $\tau_{\max }=\max \mathbb{T}>d_{\max } / 6$, it always holds that $\rho_{6 \tau_{\max }}=0$. Thus the condition $\sum_{i} C_{\mathrm{sol}}\left(\mathbb{A}_{i}, 2 k, t_{i}\left(\tau_{\max }\right), \rho_{6 \tau_{\max }}\right) \leq$ $12 \tau_{\max }$ holds, and $\hat{\tau}$ exists and satisfies condition (i).

Next we show that condition (ii) holds. Let $\left\{t_{i}^{\prime}\right\}$ be an arbitrary sequence satisfying that $\sum_{i} t_{i}^{\prime} \leq t$. Similarly to the proof of Lemma 3.3, one can show that $\sum_{i} C_{\mathrm{sol}}\left(\mathbb{A}_{i}, 2 k, t_{i}^{\prime}, \rho_{6} \hat{\tau}\right) \geq \sum_{i} C_{\mathrm{sol}}\left(\mathbb{A}_{i}, 2 k, t_{i}(\hat{\tau}), \rho_{6} \hat{\tau}\right)$, using the fact that $\sum_{i} t_{i}^{\prime} \leq t<\rho t=\sum_{i} t_{i}$. Combining with Lemma 5.9 with $\epsilon=1$, we have that

$$
\begin{aligned}
6 \sum_{i} C_{\mathrm{opt}}\left(\mathbb{A}_{i}, k, t_{i}^{\prime}, \rho_{2 \hat{\tau}}\right) & \geq \sum_{i} C_{\mathrm{sol}}\left(\mathbb{A}_{i}, 2 k, t_{i}^{\prime}, \rho_{6 \hat{\tau}}\right) \\
& \geq \sum_{i} C_{\mathrm{sol}}\left(\mathbb{A}_{i}, 2 k, t_{i}(\hat{\tau}), \rho_{6 \hat{\tau}}\right) \geq 12 \hat{\tau},
\end{aligned}
$$

whence condition (ii) follows.
LEMMA 5.11. Suppose that $\hat{\tau}$ satisfies the condition (i) and (ii) of Lemma 5.10, a $\gamma$-approximation of the weighted center-g problem induced by preclustering $\operatorname{sol}\left(\mathbb{A}_{i}, 2 k, t_{i}(\hat{\tau}), \rho_{6 \hat{\tau}}\right)$ is an $O(\gamma)$ approximation of $C_{\text {opt }}(\mathbb{A}, k, t)$.

To prove this lemma, we need the following two auxiliary lemmas.

LemmA 5.12. $2 C_{\text {opt }}\left(\mathbb{A}, k, t, \rho_{\tau}\right) \geq \sum_{i} C_{\text {opt }}\left(\mathbb{A}_{i}, k, t_{i}^{*}, \rho_{2 \tau}\right)$, where $t_{i}^{*}$ is the number of ignored nodes from $\mathbb{A}_{i}$ in the global optimum solution opt $\left(\mathbb{A}, k, t, \rho_{\tau}\right)$.

Proof. Fix a realization of the nodes. The proof mimics Lemma 3.2 for each realization. It then uses the observation that $\mathcal{L}_{\tau}\left(u_{1}, u_{2}\right)+$ $\mathcal{L}_{\tau}\left(u_{2}, u_{3}\right) \geq \mathcal{L}_{2 \tau}\left(u_{1}, u_{3}\right)$ and takes the expectation.

LemmA 5.13. If $C_{\text {opt }}\left(Z, k, t, \rho_{\tau}\right) \geq \tau$ then $C_{\text {opt }}(Z, k, t) \geq \tau / 3$.

Proof. The case of $t=0$ (no outliers) is proved in [15, Lemma 4.4]. For a general $t>0$, let $Z^{\prime} \subseteq Z$ be the set of clustered point in $\operatorname{opt}(Z, k, t)$, then $C_{\text {opt }}\left(Z^{\prime}, k, 0, \rho_{\tau}\right)=C_{\text {opt }}\left(Z, k, t, \rho_{\tau}\right) \geq \tau$, thus $C_{\text {opt }}(Z, k, t)=C_{\text {opt }}\left(Z^{\prime}, k, 0\right) \geq \tau / 3$.

Proof of Lemma 5.11. It follows from Lemma 5.12 and condition (ii) of Lemma 5.10 that

$$
2 C_{\text {opt }}\left(\mathbb{A}, k, t, \rho_{\hat{\tau}}\right) \geq \sum_{i} C_{\text {opt }}\left(\mathbb{A}_{i}, k, t_{i}^{*}, \rho_{2 \hat{\tau}}\right) \geq 2 \hat{\tau},
$$

where $t_{i}^{*}$ is the number of ignored nodes from $\mathbb{A}_{i}$ in the global optimum solution $\operatorname{opt}\left(\mathbb{A}, k, t, \rho_{\hat{\tau}}\right)$. It then follows from Lemma 5.13 that $C_{\text {opt }}(\mathbb{A}, k, t) \geq \hat{\tau} / 3$,

To simplify the notation, in the rest of the proof we shorthand $t_{i}(\hat{\tau})$ as $t_{i}$. Let $\mathbb{A}_{i}^{*} \subseteq \mathbb{A}_{i}$ be the set of nodes clustered in the global optimum solution opt $(\mathbb{A}, k, t)$. Consider "collapsing" the nodes in $\mathbb{A}_{i}^{*}$ to their corresponding centers in $\operatorname{sol}\left(\mathbb{A}_{i}, 2 k, t_{i}, \rho_{6} \hat{\tau}\right)$ while keeping the same centers in $\operatorname{sol}(\mathbb{A}, k, t)$. If a node in $\mathbb{A}_{i}^{*}$ is marked as an outlier in $\operatorname{sol}\left(\mathbb{A}_{i}, 2 k, t_{i}, \rho_{2 \hat{\tau}}\right)$ then it is not moved, and it continues to be excluded from the calculation. This movement increases the expectation of the maximum assignment by $6 \hat{\tau}+C_{\text {sol }}\left(\mathbb{A}_{i}, 2 k, t_{i}, \rho_{2} \hat{\tau}\right)$. Now consider the same process where we collapse $\mathbb{A}_{i}^{*}$ for all $i$. The total increase across the different $i$ is $6 \hat{\tau}+\sum_{i} C_{\text {sol }}\left(\mathbb{A}_{i}, 2 k, t_{i}, \rho_{6} \hat{\tau}\right)$ because the increase in $6 \hat{\tau}$ arises from distance truncation and is common. Thus we achieve a solution of cost at most

$$
\gamma\left(C_{\text {opt }}(\mathbb{A}, k, t)+6 \hat{\tau}+\sum_{i} C_{\text {sol }}\left(\mathbb{A}_{i}, 2 k, t_{i}, \rho_{6 \hat{\tau}}\right)\right) .
$$

Now consider "expanding" the nodes of $\mathbb{A}_{i}$ from the preclustering to the distribution $\mathcal{D}_{j}$. By that logic the expected maximum can increase by at most $2 \hat{\tau}+\sum_{i} C_{\text {sol }}\left(\mathbb{A}_{i}, 2 k, t_{i}, \rho_{2} \hat{\tau}\right)$, which by condition (i) of Lemma 5.10 totals to $O(\gamma \hat{\tau})=O(\gamma) \mathcal{C}_{\text {opt }}(\mathbb{A}, k, t)$. The lemma follows.

We state the main theorem for the $(k, t)$-center-g problem to conclude this section.

Theorem 5.14. For the distributed $(k, t)$-center-g problem, Algorithm 4 outputs $\operatorname{sol}(\mathbb{A}, k,(1+\epsilon) t)$ satisfying $C_{\text {sol }}(\mathbb{A}, k,(1+\epsilon) t)=$ $O(1+1 / \epsilon) \cdot C_{\text {opt }}(\mathbb{A}, k, t)$. The sites communicate a total of $\tilde{O}(s k B+$ $s \log \Delta+t I)$ bits of information to the coordinator over 2 rounds, where I is the bit complexity to encode a node. The runtime at site $i$ is $\tilde{O}\left((k+t) n_{i} \log \Delta\right)$ and the runtime at the coordinator is $\tilde{O}\left((s k+t)^{2}\right)$. 


\begin{tabular}{|c|c|c|c|c|c|c|}
\hline Objective & Approx. & Centers & Ignored & Rounds & Total Comm. & Local Time \\
\hline \multirow[t]{2}{*}{ median } & \multirow[t]{2}{*}{$O(1)$} & \multirow[t]{2}{*}{$k$} & $t$ & $\begin{array}{l}1 \\
2\end{array}$ & $\begin{array}{l}\tilde{O}((s k+s t) B) \\
\tilde{O}((s k+t) B)\end{array}$ & $\begin{array}{c}\tilde{O}\left(n_{i}^{2}\right), \tilde{O}\left(k^{2} s^{3} t^{5}\right) \\
\tilde{O}\left(n_{i}^{2}\right), \tilde{O}\left(k^{2} t^{2}(s k+t)^{3}\right)\end{array}$ \\
\hline & & & $(2+\delta) t$ & 2 & $\tilde{O}(s / \delta+s k B)$ & $\tilde{O}\left(n_{i}^{2}\right), \tilde{O}\left(s^{2} k^{7}\right)$ \\
\hline \multirow{2}{*}{$\begin{array}{l}\text { means/ } \\
\text { median }\end{array}$} & \multirow{2}{*}{$O(1+1 / \epsilon)$} & \multicolumn{2}{|c|}{$k,(1+\epsilon) t$ or $(1+\epsilon) k, t$} & $\begin{array}{l}1 \\
2\end{array}$ & $\begin{array}{c}\tilde{O}((s k+s t) B) \\
\tilde{O}((s k+t) B)\end{array}$ & $\begin{array}{c}\tilde{O}\left(n_{i}^{2}\right), \tilde{O}\left((s k+s t)^{2}\right) \\
\tilde{O}\left(n_{i}^{2}\right), \tilde{O}\left((s k+t)^{2}\right)\end{array}$ \\
\hline & & $\begin{array}{c}k \\
(1+\epsilon) k\end{array}$ & $\begin{array}{c}(2+\epsilon+\delta) t \\
(2+\delta) t\end{array}$ & 2 & $\tilde{O}(s / \delta+s k B)$ & $\tilde{O}\left(n_{i}^{2}\right), \tilde{O}\left((s k)^{2}\right)$ \\
\hline \multirow[t]{2}{*}{ center } & \multirow[t]{2}{*}{$O(1)$} & \multirow[t]{2}{*}{$k$} & $t$ & $\begin{array}{l}1 \\
2\end{array}$ & $\begin{array}{c}\tilde{O}((s k+s t) B) \\
\tilde{O}((s k+t) B)\end{array}$ & $\begin{array}{l}\tilde{O}\left((k+t) n_{i}\right), \tilde{O}\left((s k+s t)^{2}\right) \\
\tilde{O}\left((k+t) n_{i}\right), \tilde{O}\left((s k+t)^{2}\right)\end{array}$ \\
\hline & & & $(2+\delta) t$ & 2 & $\tilde{O}(s / \delta+s k B)$ & $\tilde{O}\left(n_{i}^{2}\right), \tilde{O}\left((s k)^{2}\right)$ \\
\hline $\begin{array}{c}\text { uncertain } \\
\text { median/ } \\
\text { means/ } \\
\text { center-pp }\end{array}$ & \multicolumn{5}{|c|}{ as in the regular case above } & $+O\left(n_{i} T\right)$, unchanged \\
\hline center-g & $\begin{array}{c}O(1+1 / \epsilon) \\
O(1)\end{array}$ & $k$ & $\begin{array}{c}(1+\epsilon) t \\
t\end{array}$ & $\begin{array}{l}2 \\
1 \\
\end{array}$ & $\begin{array}{c}\tilde{O}(s k B+t I+s \log \Delta) \\
\tilde{O}(s(k B+t I) \log \Delta)\end{array}$ & $\begin{array}{c}\tilde{O}\left(n_{i}^{2} \log \Delta\right), \tilde{O}\left((s k+t)^{2}\right) \\
\tilde{O}\left((k+t) n_{i} \log \Delta\right), \tilde{O}\left(s^{2}(k+t)^{2}\right)\end{array}$ \\
\hline
\end{tabular}

Table 2: Our results. $T$ denotes the runtime to compute 1-median/mean of a node distribution, $I$ is the information encoding a node in the uncertain data case, $B$ the information encoding a point and $\Delta$ the ratio between the maximum pairwise distance and the minimum pairwise distance in the dataset.

Proof. The claim on approximation ratio follows from Lemma 5.11. To determine $\hat{\tau}$, the communication cost increases by a factor of $\log \Delta$; to send the preclustering solutions, the communication cost for sending the outliers increases by a factor of $I$. The runtime follows from Lemma 5.9 with an increase of a factor of $\log \Delta . \quad \square$

We remark that the dependence on $\log \Delta$ can be removed with another pass where each site computes a $\tau_{i}$ using binary search The discussion is omitted in the interest of simplicity.

Other results claimed in Table 2 follow from analogous amendments to Theorem 3.8.

\section{REFERENCES}

[1] Charu C. Aggarwal. A survey of uncertain data clustering algorithms. In Dato Clustering: Algorithms and Applications, pages 457-482. 2013.

[2] Maria-Florina Balcan, Steven Ehrlich, and Yingyu Liang. Distributed k-means and k-median clustering on general communication topologies. In Proceedings of NIPS, pages 1995-2003, 2013

[3] Moses Charikar and Sudipto Guha. Improved combinatorial algorithms for the facility location and k-median problems. In Proceedings of FOCS, pages 378-388, 1999.

[4] Moses Charikar, Samir Khuller, David M. Mount, and Giri Narasimhan. Algorithms for facility location problems with outliers. In Proceedings of SODA, pages 642-651, 2001.

[5] Jiecao Chen, He Sun, D. Woodruff, and Qin Zhang. Communication-optimal distributed clustering. In Proceedings of NIPS, 2016.

[6] Ke Chen. A constant factor approximation algorithm for $k$-median clustering with outliers. In Proceedings of SODA, pages 826-835, 2008.

[7] Michael B. Cohen, Sam Elder, Cameron Musco, Christopher Musco, and Madalina Persu. Dimensionality reduction for $k$-means clustering and low rank approximation. In Proceedings of STOC, pages 163-172, 2015.

[8] Graham Cormode and Andrew McGregor. Approximation algorithms for clustering uncertain data. In Proceedings of PODS, pages 191-200, 2008.

[9] Graham Cormode, S Muthukrishnan, and Wei Zhuang. Conquering the divide: Continuous clustering of distributed data streams. In Proceedings of ICDE, pages 1036-1045. IEEE, 2007

[10] M. E. Dyer. On a multidimensional search technique and its application to the euclidean one centre problem. SIAM 7. Comput., 15(3):725-738, 1986.

[11] Alina Ene, Sungjin Im, and Benjamin Moseley. Fast clustering using mapreduce In Proceedings of SIGKDD, pages 681-689, 2011.

[12] Dan Feldman and Leonard J. Schulman. Data reduction for weighted and outlierresistant clustering. In Proceedings of SODA, pages 1343-1354, 2012.

[13] Teofilo F. Gonzalez. Clustering to minimize the maximum intercluster distance. Theor. Comput. Sci., 38:293-306, 1985.

[14] Sudipto Guha, Adam Meyerson, Nina Mishra, Rajeev Motwani, and Liadan O'Callaghan. Clustering data streams: Theory and practice. IEEE Trans. on Knowl. and Data Eng., 15(3):515-528, 2003.
[15] Sudipto Guha and Kamesh Munagala. Exceeding expectations and clustering uncertain data. In Proceedings of PODS, pages 269-278, 2009.

[16] Sungjin Im and Benjamin Moseley. Brief announcement: Fast and better distributed mapreduce algorithms for $k$-center clustering. In Proceedings of SPAA, pages $65-67,2015$

[17] Kamal Jain and Vijay V. Vazirani. Approximation algorithms for metric facility location and $\mathrm{k}$-median problems using the primal-dual schema and lagrangian relaxation. F. ACM, 48(2):274-296, 2001.

[18] Yingyu Liang, Maria-Florina Balcan, Vandana Kanchanapally, and David P. Woodruff. Improved distributed principal component analysis. In Proceedings of NIPS, pages 3113-3121, 2014.

[19] Gustavo Malkomes, Matt J. Kusner, Wenlin Chen, Kilian Q. Weinberger, and Benjamin Moseley. Fast distributed $k$-center clustering with outliers on massive data. In Proceedings of NIPS, pages 1063-1071, 2015.

[20] Dan Suciu, Dan Olteanu, R. Christopher, and Christoph Koch. Probabilistic Databases. Morgan \& Claypool Publishers, 1st edition, 2011.

[21] Haitao Wang and Jingru Zhang. One-dimensional $k$-center on uncertain data. Theoretical Computer Science, 602:114 - 124, 2015.

\section{A THE FULL SET OF OUR RESULTS}

We summarize the full set of our results in Table 2. Besides the main results that already appear in Table 1 , all the 1-round results in Table 2 basically follow from setting $t_{i}=t$ for all sites $i$. The results for $(k, t)$-median/means that ignore $(2+\delta) t$ or $(2+\epsilon+\delta) t$ points basically follow from Theorem 3.8, where for $(k, t)$-median with $k$ centers (unicriterion) we need to apply again the 1-round result, and for $(k, t)$-median/means with $(1+\epsilon) k$ centers we simply use the second inequality of Theorem 3.1 instead of the first one at the final clustering step at the coordinator. The result for $(k, t)$-center that ignore $(2+\delta) t$ points is due to the following modifications on Algorithm 4: sites do not send the total $(1+\delta) t$ local outliers to the coordinator, and thereafter the coordinator performs the second level clustering with (another) $t$ outliers, we have $(2+\delta) t$ outliers in total. 